\title{
ComSem: Digitization and Semantic Annotation of Comic Books
}

\author{
Joachim Van Herwegen, Ruben Verborgh, and Erik Mannens \\ Ghent University - imec - IDLab \\ Sint-Pietersnieuwstraat 41, B-9000 Ghent, Belgium \\ joachim.vanherwegen@ugent.be
}

\begin{abstract}
The popularity of digital comic books keeps rising, causing an increase in interest from traditional publishers. Digitizing existing comic books can require much work though, since older comic books were made when digital versions were not taken into account. Additions such as digital panel segmentation and semantic annotation, which increase the discoverability and functionality, were only introduced at a later point in time. To this end, we made ComSem: a tool to support publishers in this task by automating certain steps in the process and making others more accessible. In this paper we present our demo and how it can be used to easily detect comic book panels and annotate them with semantic metadata.
\end{abstract}

Keywords: digital publishing, EPUB3, comic book

\section{Introduction}

Digital comic books are becoming more and more popular. Comic book sales keep increasing every year, both physically and digitally ${ }^{1}$. Because of this, more and more publishers are interested in entering this digital market, both with their new releases and their older backlog. Unfortunately, most of their backlog was designed in a time when there was no demand for digital comics. This means that at most they might have an archive of images corresponding to the complete pages of the comics. These images are not enough to create a pleasant reading experience: it is for example often infeasible to display an entire comic page on a mobile device, meaning additional steps have to be taken to create a pleasant reading experience.

Publishers are also often not that aware of how they can annotate their comic books with semantic data or what advantages this offers. Adding meta-information to comics can greatly increase their discoverability and usability [5].

In this paper we showcase our Comic Semantifier, ComSem, which assists publishers in their digital production pipeline, starting from a set of images and ending with a semantically annotated EPUB3 file. The demo itself can be found

\footnotetext{
${ }^{1}$ http://www. cnbc . com/2016/06/05/comic - books - buck - trend - as - print - and - digital sales - flourish . html
} 
at http://comsem. demo.idlab.technology $/^{2}$. ComSem was made in cooperation with actual comic book publishers and is based on their workflow and needs.

\section{Related Work}

\subsection{Digital comic book tools}

There are many tools available for creating digital comic books ${ }^{3}$, but most of these focus on creating the comic itself in their tool, instead of adapting an existing comic book to a digital format, making them less suited for publishers looking to digitize their existing backlog. Others still require much manual labour to specify where all the panels are located on a page. There is also very little focus on the semantic aspect of the comic books, providing publishers with no option to embed this in their work, which limits their potential as described in Section 2.3.

\section{$2.2 \quad$ EPUB3 and RDFa}

EPUB3 [2] is a standard for the digital publication of e-books. It consists of an archive of HTML files, describing both the content and metadata, combined with relevant assets. Although the format was originally designed for e-books, work has been put into extending the format for digital comic books ${ }^{4}$.

$\mathrm{RDFa}^{5}$ is a format designed to embed RDF data into HTML pages. Since EPUB3 is a collection of HTML, RDFa can also be used to enrich EPUB3 files, which is how ComSem annotates comic books.

\subsection{Metadata}

Research has shown that the addition of metadata to digital publications can have several positive impacts, such as increasing discovery, supporting dynamic content and providing semantic search $[1,3,5]$.

Discoverability plays a big role in digital publications. With a surplus of offerings available, it becomes harder to stand out in the crowd. In his study on optimizing digital publications, Wischenbart [5] explains the importance of metadata to improve the chances of people discovering your work.

An example tool showcasing the advantages of metadata is the digital authoring environment created by De Meester et al. [1], supporting content creators by providing easy to use tools for enhancing their text with semantic metadata. Similarly, Heyvaert et al. have created a tool making use of comic book metadata to improve the user experience [3] by allowing them to apply specific filters on comic book collections.

\footnotetext{
2 An example comic can be found at http://comsem.demo.idlab.technology/dnd.html? comic=1 (Saving changes and NER are disabled on this specific comic for demo reasons).

3 http ://networkcultures . org/longform/2016/11/21/digital - comics - harder - better - 


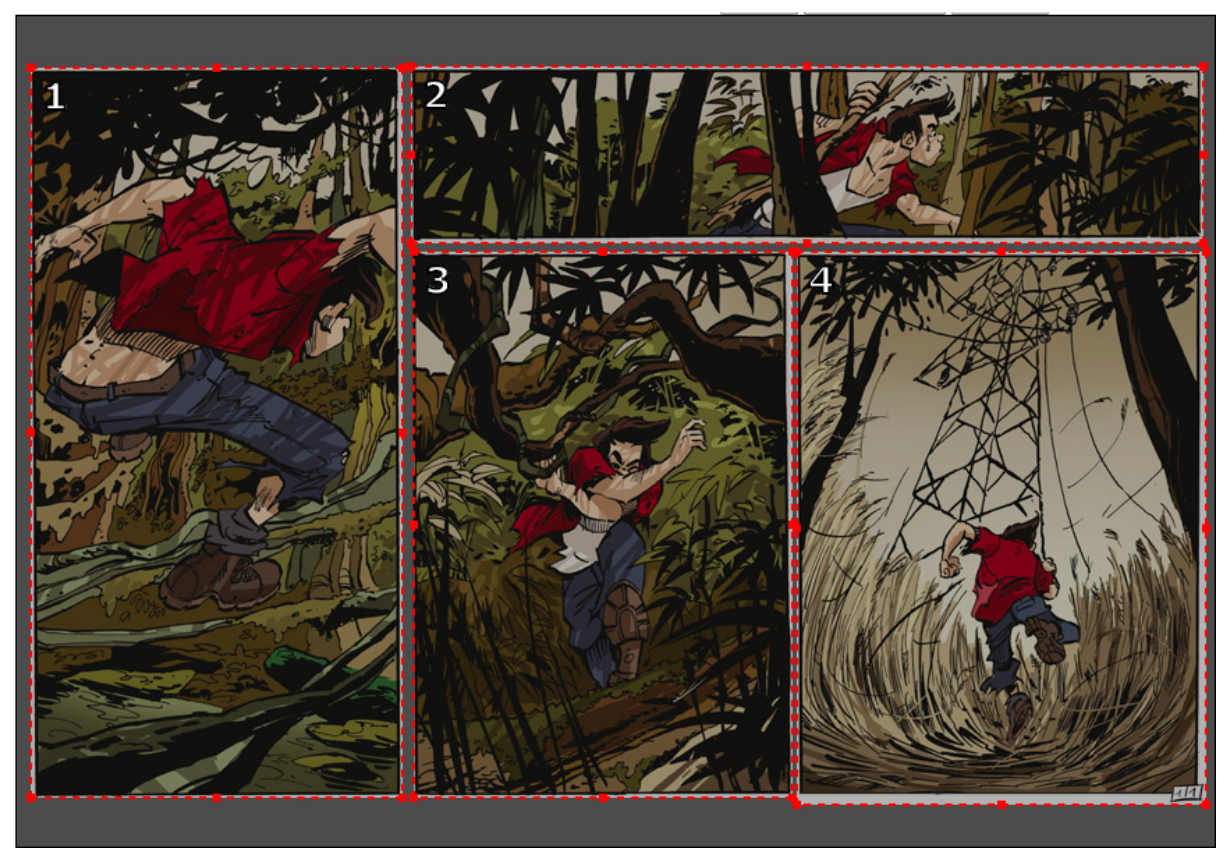

Fig. 1. Automated panel detection. The borders, number of panels, and order of the panels can easily be changed by right-clicking the panels.

\section{Comic book digitization}

ComSem starts from a set of digital images, and ends with a valid EPUB3 containing all the relevant metadata. The user begins by uploading a set of images, which can be done by dragging them to the relevant area, after which ComSem automatically detects the specific panels on each of them. Afterwards these can be annotated with additional semantic metadata. Then, once the user has finished, the result can be exported as EPUB3.

\subsection{Automated panel detection}

Our implementation is currently based on a flood-fill implementation, making use of the opencV ${ }^{6}$ library, with additional support for detecting overlapping panels, similar to the method described by Ho et al. [4]

Figure 1 shows an example of the panel detection done by ComSem after uploading a page. As can be seen, the detection is not completely correct. This can be caused by panel lines not being $100 \%$ straight, or by having components extending outside of the panel, as is the case for the bottom-right panel in this figure. The handles on the sides of the panels allow the user to manually fine-tune the panel positioning in the cases where this is deemed necessary.

\footnotetext{
$\overline{{ }^{6} \text { http://opencv.org/ }}$
} 
The order of the panels is also determined automatically, based on their relative positioning. This order can easily be changed by right-clicking on the panels in the order they should appear.

Should the number of detected panels be incorrect, new panels can quickly be added by double-clicking, while superfluous panels can be deleted by doubleclicking them while holding the ALT key.

\subsection{Semantic annotation}

During the creation of an EPUB3, we provide the user with ample opportunity to enrich the content with RDFa, using the tools described below. In ComSem, we have mostly focused on metadata such as the characters appearing in the story, using metadata to indicate their appearance on specific panels, and transcribing the textual contents of a panel, allowing for translations to be present. But we also support custom RDF tags, making it possible to describe other components.

Annotation tools There are two types of metadata that can be added in ComSem: panel-specific and global. Panel-specific metadata pertains to the events depicted in a specific panel, while global metadata describes information corresponding to the entire comic book, such as the author.

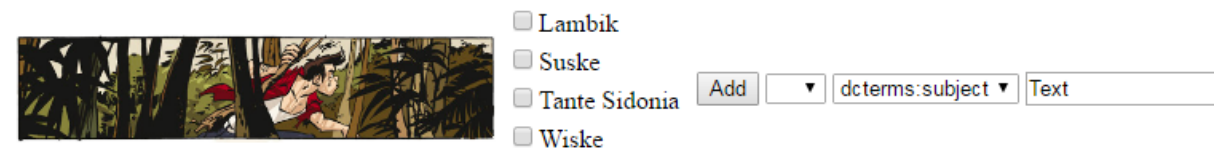

Fig. 2. Panel metadata. The panels are automatically generated based on the selection made before.

In the panel-specific tab (Figure 2), the check-boxes can be used to indicate the presence of a specific character in the given panel. This metadata gets stored using the schema:about ${ }^{7}$ tag, while the text-boxes provide the user with the option of adding additional non-character tags, using, among others, schema: text and schema:url. Users can also update the list of characters and metadata to choose from, which is not pictured here.

Besides the panel editor, there is also the global metadata editor (Figure 3). There the user can add global metadata, such as ISBN, title, and author. A short description of the comic book can also be written in the given text field. This description can then be used for enriching the comic with additional metadata. When the Enrich button is pressed, the description text is sent to a Named Entity Recognition (NER) engine, after which all recognized entities are added using the schema: about tag. The NER engine is currently configured for the Dutch language,

\footnotetext{
7 https://schema.org/
} 


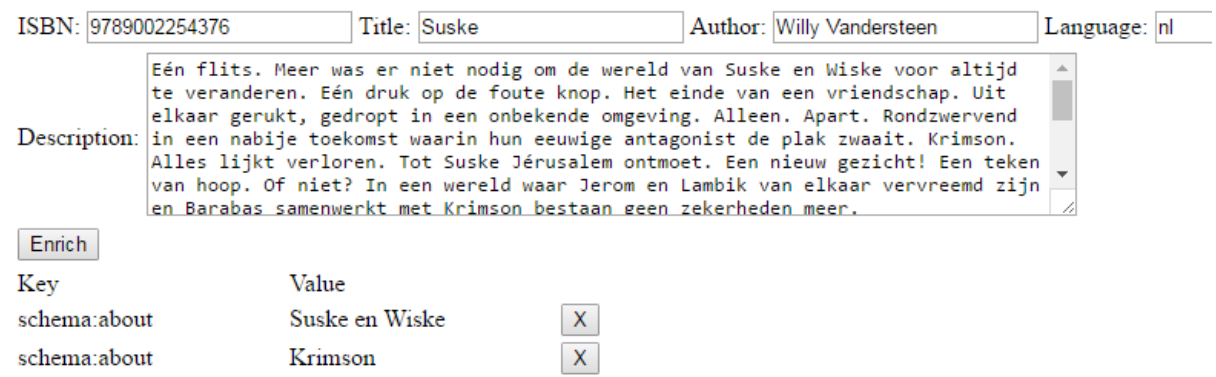

Fig. 3. Global metadata. New tags can easily be added or removed.

but will be adapted to depend on the value of the language tag provided by the user. There is also the option for users to add extra tags, should more specific predicates be required. All this metadata is then added to the EPUB3 file once it is generated.

\section{Conclusion}

ComSem supports publishers in digitizing and annotating their existing backlog of comic books. It also provides them with the opportunity to add metadata in those comic books, enriching their content and providing the possibility of integrating with new applications to improve the user experience. It was made in such a way that it can easily be used by someone with no Semantic Web background, while still having full RDF support.

Due to the automated panel detection, the digitization process can be sped up, since only minor adjustments are required to perfectly mark the panel locations, after which the comic book can immediately be converted to a valid EPUB3 file.

\section{References}

1. De Meester, B., De Nies, T., Ghaem Sigarchian, H., Vander Sande, M., Van Campen, J., Van Impe, B., De Neve, W., Mannens, E., Van de Walle, R.: A Digital-First Authoring Environment for Enriched e-Books using EPUB 3. Information Services and Use pp. 259-268 (2014), http://dx.doi.org/10.3233/ISU- 140748

2. Gylling, M., McCoy, W., Garrish, M.: Epub publications 3.0. Recommended specification, IDPF (2011), http://www.idpf.org/epub/30/spec/epub30-publications.html

3. Heyvaert, P., De Nies, T., Van Herwegen, J., Vander Sande, M., Verborgh, R., De Neve, W., Mannens, E., Van de Walle, R.: Using epub 3 and the open web platform for enhanced presentation and machine-understandable metadata for digital comics. In: ELPUB 2015. pp. 37-46 (2015)

4. Ho, A.K.N., Burie, J.C., Ogier, J.M.: Panel and speech balloon extraction from comic books. In: 2012 10th IAPR International Workshop on Document Analysis Systems. pp. 424-428 (2012), http://dx.doi.org/10.1109/DAS.2012.66

5. Wischenbart, R.: Global eBook: A report on market trends an developments. O'Reilly Media (2014) 